\title{
Effect of Silica Modification on Charge Trapping Behavior of PP blend/Silica Nanocomposites
}

\author{
Amirhossein Mahtabani ${ }^{1}$, Xiaozhen $\mathrm{He}^{1}$, Ilkka Rytöluoto ${ }^{2,3}$, Kari Lahti ${ }^{2}$, Mika Paajanen ${ }^{3}$, Eetta Saarimäki ${ }^{3}$ \\ Rafał Anyszka ${ }^{1}$, Wilma Dierkes ${ }^{1}$, Anke Blume ${ }^{1}$ \\ ${ }^{1}$ University of Twente, Faculty of Engineering Technology, Department of Mechanics of Solids, Surfaces \& Systems (MS3), \\ Chair of Elastomer Technology and Engineering, Enschede, The Netherlands \\ ${ }^{2}$ Tampere University, Laboratory of Electrical Energy Engineering, Tampere, Finland \\ ${ }^{3}$ VTT Technical Research Centre of Finland Ltd, Tampere, Finland
}

\begin{abstract}
Various dielectric nanocomposite materials are studied in the frame of the European Commission funded project GRIDABLE. This project has the aim to develop DC cable extruded insulation and medium and low voltage DC capacitor films exhibiting enhanced performance with respect to presently used materials. The nanocomposites intended for cable applications are based on polypropylene (PP) blends filled with surface modified nano-silica particles. The surface modification is carried out via the state-of-the-art solution method using a polar silane as the modifying agent. Thermally Stimulated Depolarization Current (TSDC) measurements were carried out in order to study the charge trapping behavior of the nanocomposite samples. TSDC results indicate that the addition of the treated nano-silica, for most cases, reduces the density of the deep traps significantly. While more detailed studies are necessary, these results imply that the depth and the density of the deep trap states is profoundly influenced by the level of the silica modification i.e. the amount of the grafted silane on the silica surface.
\end{abstract}

Keywords: Silica surface modification, PP blend/Silica dielectric nanocomposites, Charge trapping properties, TSDC, High voltage cable insulation

\section{INTRODUCTION}

A European project called GRIDABLE (Polymer Nanocomposite Insulation Material Enabling Integration of Renewables and DC Storage Technologies in the AC Energy Grid) was inaugurated under the HORIZON 2020 framework co-funded by the European Community. Various partners, both academic and industrial, are working together within this project, aiming to develop a new generation of nanostructured polymer-based materials suitable for high voltage DC (HVDC) cable insulators and capacitors. The goal of GRIDABLE is to develop polypropylene-based nanocomposites with proper nanoparticle functionalization and dispersion quality. This novel material can introduce significant improvements to the state of the art DC cable and film capacitor insulations.

The incorporation of nanoparticles to dielectric materials, in order to improve their insulating properties, was studied extensively in the recent years [1-3]. Nanodielectric composites containing filler particles of size lower than $100 \mathrm{~nm}$ in one dimension, may exhibit significant improvements in e.g. dielectric strength and voltage endurance [4]. These improvements can be addressed to various phenomena, which mainly stem from the high interfacial area implemented by nanofillers incorporated to a polymer matrix [5] as well as the alteration of the features of the polymer/filler interphase [4]. Upon changes of the type and surface characteristics of the filler, the polymer/filler interphase region can influence crystallinity [6], molecular mobility [7] and entanglement density [8]. These changes in the nanoparticles' surface properties can contribute to further improvements of the dielectric properties of the nanocomposites filled with them. Changing the surface properties of the nanoparticles is possible through surface modification that is substituting the existing chemical structure on the particles' surface with a certain functionality tailored for a specific application.

Surface modification of nanofillers may enhance the dielectric properties of the nanocomposites by improving their dispersion in a polymer matrix. Since the nanofiller particles act as electrical defect centers in polymers [9], they can distort and enhance the local electric field in their vicinity, which causes a decrease in the dielectric breakdown strength as compared to the neat polymer. The extent of this local field distortion and enhancement is not only a factor of the difference in permittivity or conductivity between the particles and the polymer matrix, but also of the size of the particles, their aggregation and agglomeration [1]. Therefore, improving the dispersion of the nanofillers in a nanodielectric material can result in improvement of the dielectric breakdown strength.

Another factor that can lead to early electrical breakdown of a nanocomposite, is poor adhesion between the particles and polymer chains due to chemical incompatibility [10]. Chemical incompatibility could result in formation of small voids at the polymer/filler interface and thus decrease the dielectric breakdown strength. Hence, surface modification performed in order to bond/bind the particle surface to the polymer matrix, can increase the breakdown strength and reduce the dielectric losses [11].

Another way in which surface modification of the nanoparticles can influence the dielectric properties of the 
nanodielectrics is alteration of the electronic features at the interface between the coated particles and the polymer matrix [1]. Siddabattuni et al. [12], reported a significant reduction in leakage currents and dielectric losses and improvement in dielectric breakdown strengths, when electropositive phenyl, thus electron-withdrawing functional groups, were located at the polymer-particle interface. Accordingly, it can be speculated that introduction of polar functional groups to the surface of the nanoparticles can induce this sort of electronic behavior as the dipoles at the particle/polymer interface may affect charge carrier transport and trapping [13].

Space charge distribution is a direct outcome of combined trapping/detrapping and transport behavior of charges in insulating polymers and varies with trap density, trap depth distribution, and charge carrier mobility [14]. While the space charge phenomena can possibly be controlled by changing the morphology and crystallinity of the polymer, space charge distribution can be influenced by grafting functional groups onto the nanofiller particle surface, influencing charge mobility through the introduction of shallow trap sites [15] or reducing the number of charges trapped in deep traps [1] in the filler/polymer interaction zones.

In this study, silica nanoparticles were modified with a polar modifying agent, and compounded with PP based polymer blends. The aim of surface modification was to improve the trapping/detrapping behavior of the PP blend/silica nanocomposites. Firstly, the modification was proven by using Thermogravimetric Analysis (TGA). Subsequently, the influence of silica modification on the charge trapping behavior of the aforementioned nanocomposites was studied via Thermally Stimulated Depolarization Current (TSDC). Nevertheless, this paper is based on preliminary results of the study, and further elaborations are planned to be done in future publications.

\section{EXPERIMENTAL WORK}

\section{A. SILICA MODIFICATION}

A fumed silica grade with high purity and low moisture content was selected for this study. The silica surface modification was carried out via the state-of-the-art solution method using a polar silane as the modifying agent under different reaction conditions. Further specification of these materials is at this moment not possible due to intellectual property reasons. The details of each modification reaction for each modified silica sample is depicted in Table 1. The modification was carried out in a glass round bottom flask, immersed in an oil heating bath, equipped with a mechanical stirrer, cooler and thermometer. The silica, silane and additional water were mixed with toluene, which was the liquid medium of the reaction.

The polar silane used in this study is prone to alter the silicapolymer interphase which, as a consequence, can change the dielectric behavior of the nanocomposites. It is assumed that by the introduction of this polar functionality to the silica surface, charge trapping properties of the dielectric material undergo meaningful changes, which might induce beneficial influences on the ultimate insulation characteristics of the nanocomposite materials.

TABLE I

\begin{tabular}{|c|c|c|c|}
\hline $\begin{array}{l}\text { Sample } \\
\text { Number }\end{array}$ & $\begin{array}{c}\text { Temperature } \\
\left({ }^{\circ} \mathrm{C}\right)\end{array}$ & $\begin{array}{l}\text { Time } \\
\text { (hour) }\end{array}$ & $\begin{array}{c}\text { Added Water } \\
\text { (gram) }\end{array}$ \\
\hline 0 & \multicolumn{3}{|c|}{ Untreated Silica } \\
\hline 1 & 80 & 24 & 1.8 \\
\hline 2 & 20 & 1 & 1.8 \\
\hline 3 & 80 & 1 & 0.0 \\
\hline 4 & 20 & 24 & 1.8 \\
\hline 5 & 80 & 1 & 1.8 \\
\hline
\end{tabular}

\section{B. PREPARATION OF THE NANOCOMPOSITES}

The nanocomposites studied in this work are based on polypropylene (PP) blends filled with surface modified nanosilica particles. The specimens were prepared by addition of $1 \mathrm{w} \%$ of different silica samples to the polymer matrix in a twin-screw micro extruder, and subsequently, injected into a square mold using a mini-injection molding machine. The dimensions of the mold were $260 \times 260 \times 0.5 \mathrm{~mm}$. The resulting nanocomposite (NC) samples were named according to the silica sample used. For instance, $\mathrm{NC}_{-} 1$ is nanocomposite filled with silica sample number 1 .

\section{THERMAL GRAVIMETRIC ANALYSIS (TGA)}

Thermal gravimetric analysis was performed on the modified silica samples in order to quantify the level to which the modification process is successful. This method involves heating up the silica powder from room temperature to $850{ }^{\circ} \mathrm{C}$ with a rate of $20^{\circ} \mathrm{C} / \mathrm{min}$ while measuring the weight loss of the sample. The weight loss between $300-850^{\circ} \mathrm{C}$ can be attributed to the thermal decomposition of this silane coupling agent chemically bonded to the silica surface [16]. Fig. 1 demonstrates the results of this measurement as the weight loss percentage over temperature.

\section{THERMALLY STIMULATED DEPOLARIZATION CURRENT (TSDC)}

Thermally Stimulated Depolarization Current (TSDC) measurements were carried out in order to study the charge trapping behavior of the nanocomposites. Circular $\mathrm{Au}$ electrodes (100 nm in thickness) were deposited on both sides of the sample films by e-beam evaporation under high vacuum. The samples were first heated up to $70{ }^{\circ} \mathrm{C}$, and subjected to a 3 $\mathrm{kV} / \mathrm{mm} \mathrm{DC}$ electric field for 20 minutes under isothermal conditions. The samples were then cooled down to $-50{ }^{\circ} \mathrm{C}$ while the poling voltage was still on. Subsequently, the samples were short-circuited and linearly heated up to $140{ }^{\circ} \mathrm{C}$ at 3 ${ }^{\circ} \mathrm{C} / \mathrm{min}$, while the depolarization current was monitored by a highly sensitive ammeter.

\section{RESULTS AND DISCUSSION}

TGA results, depicted in Fig. 1, show a higher weight loss for the modified silicas compared to the untreated one, which is an 
indication of the successful modification of the silica surface. Different reaction conditions were utilized to achieve different levels of silane modification. The values of weight loss above $300{ }^{\circ} \mathrm{C}$ are listed in Table 2 . It can be seen that a range of weight losses, from ca. $3 \%$ to $6 \%$, is achieved by altering the reaction conditions.

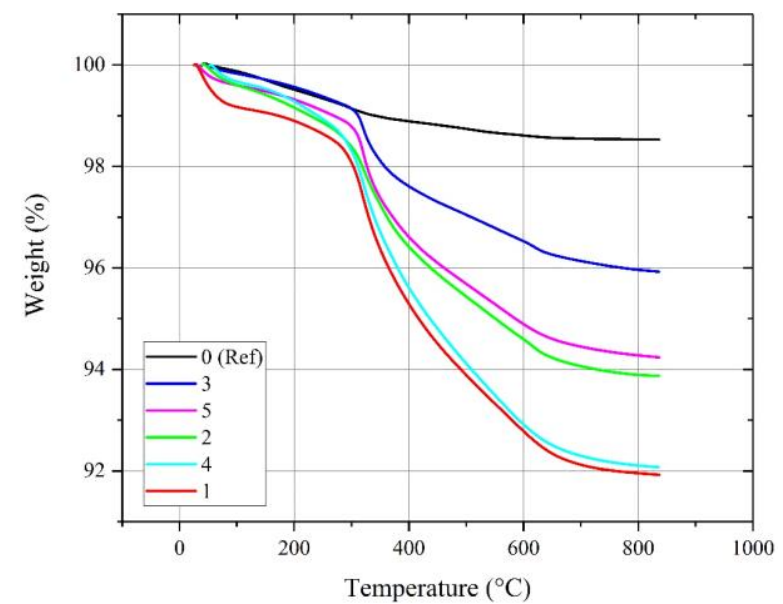

Fig. 1. TGA results for the modified silica samples compared to the untreated silica.

Nevertheless, altering the modification reaction conditions can change not only the thickness of the deposited layer, but also its morphology, wettability and extent of silane hydrolysis. Each of the aforementioned factors can affect the interfacial properties between the fillers and the polymer chains which can lead to different dielectric behavior of the nanocomposites.

Table II

TGA Weight Loss Values at Temperatures above $300{ }^{\circ} \mathrm{C}$.

\begin{tabular}{c|c}
\hline $\begin{array}{c}\text { Sample } \\
\text { Number }\end{array}$ & $\begin{array}{c}\text { TGA Weight Loss } \\
(\%)\end{array}$ \\
\hline $\boldsymbol{0}$ & 0.61 \\
$\mathbf{1}$ & 6.16 \\
$\mathbf{2}$ & 4.52 \\
$\mathbf{3}$ & 3.21 \\
$\mathbf{4}$ & 6.23 \\
$\mathbf{5}$ & 4.55 \\
\hline
\end{tabular}

Fig. 2 shows the TSDC plots of all the silica filled samples as well as of the unfilled polymer (UP) as a secondary reference. In principle, the TSDC spectra are attributable to the relaxation of (dipolar) polarization and accumulated space charge formed during the isothermal polarization phase. It is remarked that the short-circuit TSDC is a net sum of both homo- and heteropolar discharge current components, and hence, the integrated charge represents the net charge. For most semi-crystalline and weakly polar polymers, such as PP, the high temperature TSDC spectrum above the glass transition temperature is mainly associated with the relaxation of trapped space charge. It is also remarked that this high-temperature space charge relaxation can be influenced by thermal transitions (such as softening/melting) of a single phase in heterophase polymer blend systems. To a first approximation, the TSDC peak temperature and intensity can be attributed to the depth and density of charge traps, respectively, as is done in the following analysis.
The results in Fig. 2 indicate that upon incorporation of the treated nanosilica the TSDC spectra become more complex and a significant shift of the main TSDC peak towards higher temperatures occurs, being indicative of the trap becoming deeper. For the samples with the polar functionality on the filler surface a new (deep) trap emerges in the $93-97{ }^{\circ} \mathrm{C}$ range, while a smaller side peak is observed around the same temperature $\left(\sim 78{ }^{\circ} \mathrm{C}\right)$ as the intrinsic TSDC peaks of the two reference samples (UP and NC_0). Comparing NC_3 and NC_4 with the lowest and the highest levels of silica modification, respectively, it can be observed that the location of the new deep trap peak is shifted to higher temperatures. Moreover, the intensity of the TSDC peak is seen to vary significantly depending on the type of treated silica, with some of the samples exhibiting (apparent) reduction in the main peak current intensity; this may be related to (i) reduction of the density of the trap states or (ii) more uniform distribution of the space charge in the specimen (hence leading to smaller measurable external current in the TSDC experiment). While more detailed studies are necessary, these results imply that the depth and the density of the newly introduced trap is profoundly influenced by the level of the modification i.e. the amount of the grafted silane on the silica surface.

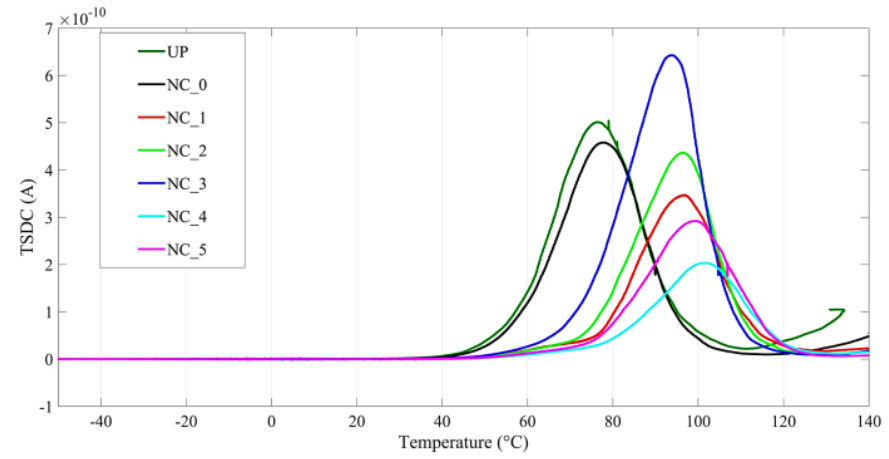

Fig. 2. TSDC spectra of injection molded PP blend samples.

The above trend is however not entirely dominant as there are some repeatable exceptions. For instance, samples NC_1 and NC_4 contain silica samples number 1 and 4, respectively, which have more or less equal TGA weight losses. However, for $\mathrm{NC}_{-} 4$, the deep trap peak is more suppressed compared to NC_1. It is the same for samples NC_2 and NC_5. In other words, a possible explanation is that the depth and density of the traps are not only a function of the thickness of the silane layer, but also of other parameters, such as filler dispersion, silane layer morphology, micro-structure and crystallization characteristics of the compound.

In order to gain more insight into the results above, the areas under current vs. time curves during polarization and depolarization were calculated, providing an indication of how much charge was injected into or released from the sample, respectively. Fig. 3 demonstrates the calculated amount of charges for each sample during isothermal polarization, isothermal polarization + freezing and depolarization. Fig. 3 can be interpreted from different points of view. Firstly, it can be seen that for all cases the amount of charge injected during 
the isothermal polarization period more or less remains in the sample as a result of freezing. Secondly, the amount of charge released during the thermally stimulated depolarization phase is much lower than that injected during polarization. While the latter is generally to be expected for TSDC experiments-in particular for relatively thick specimens for which the depth of the injected charge layer is likely small in comparison to film thickness[17] - it seems that the above effect is significantly reduced upon incorporation of modified nanosilica. Fig. 4 shows the values of released charges relative to the amount of the injected charges for these samples. As it can be seen, for the reference samples UP and NC_0, only \%19 and \%17 of the injected charges are released during depolarization, whereas, in case of samples with highly modified silica e.g. NC_1, this value increases to \%55. This can be an indication of lower remaining accumulated space charge for the samples with modified silica.

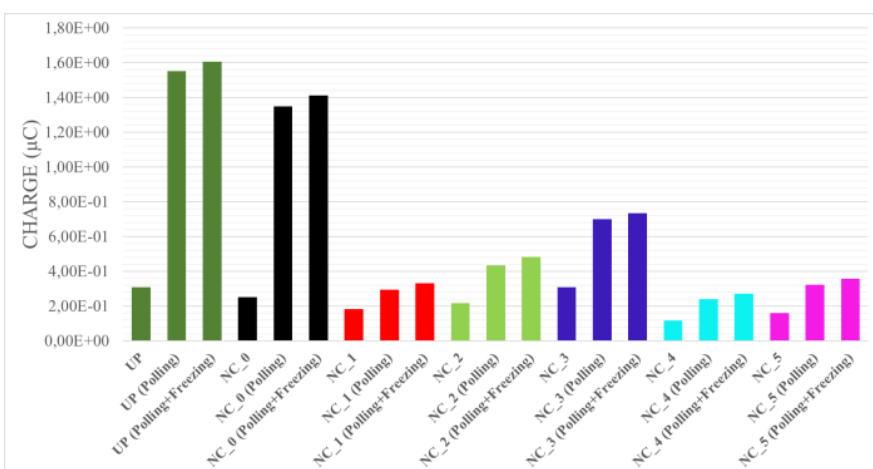

Fig. 3. Amount of charge released during thermally stimulated depolarization vs. the amount of injected charge during the polarization phase.

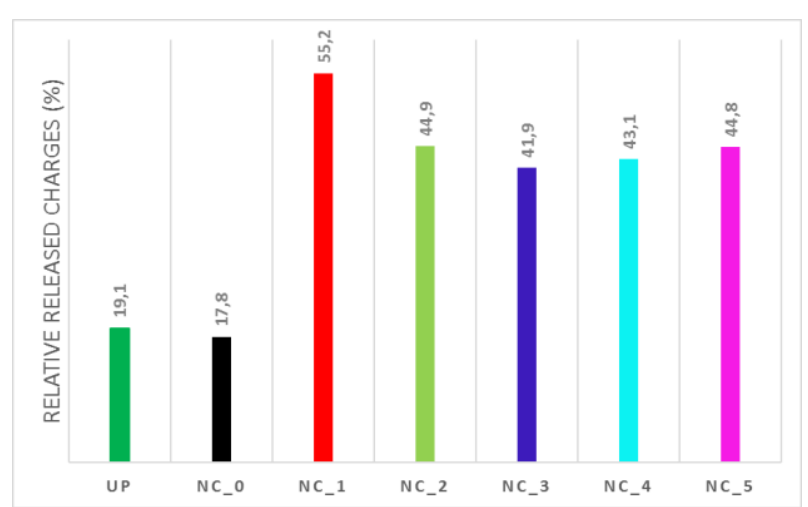

Fig. 4. Values of released charges relative to the amount of the injected charges

\section{CONCLUSIONS}

Based on the results presented in this report, modifying the surface of the silica nanoparticles have a profound effect on the charge trapping properties of the PP blend/silica nanocomposites. Upon modification, the polar functional groups added to the silica surface, alter the electronic features of the nanoparticles in such a way that the deep charge traps are suppressed and new and deeper traps are introduced. It can be seen that increasing the amount of the grafted silane on the silica surface results in more pronounced suppression of the deep traps. However, there are exceptions. Some of the samples with the same modification levels behave differently during the TSDC measurement. This could be due to the fact that altering the modification reaction conditions changes not only the amount of grafted silane, but also the grafted layer morphology, accessible surface area, wettability and extent of silane hydrolysis, each of which can affect the interfacial properties between fillers and the polymer chains leading to different dielectric behavior of the nanocomposites. In order to get a clearer image of the functionalization of the silica and its effect on the charge trapping behavior of dielectric nanocomposites, studying the aforementioned factors seems to be interesting for future studies.

\section{ACKNOWLEDGMENT}

This project has received funding from the European Union's Horizon 2020 research and innovation program under grant agreement No. 720858. The authors also would like to thank the ECIU Researcher Mobility Fund for supporting this research work.

\section{REFERENCES}

[1] M. Dongling et al., "Influence of nanoparticle surface modification on the electrical behaviour of polyethylene nanocomposites," Nanotechnology, vol. 16, no. 6, p. 724, 2005.

[2] X. Huang, P. Jiang, and Y. Yin, "Nanoparticle surface modification induced space charge suppression in linear low density polyethylene," Applied Physics Letters, vol. 95, no. 24, p. 242905, 2009.

[3] X. Huang, Y. Zheng, P. Jiang, and Y. Yin, "Influence of nanoparticle surface treatment on the electrical properties of cycloaliphatic epoxy nanocomposites," IEEE Transactions on Dielectrics and Electrical Insulation, vol. 17, no. 2, pp. 635-643, 2010.

[4] R. C. Smith, C. Liang, M. Landry, J. K. Nelson, and L. S. Schadler, "The mechanisms leading to the useful electrical properties of polymer nanodielectrics," IEEE Transactions on Dielectrics and Electrical Insulation, vol. 15, no. 1, pp. 187-196, 2008.

[5] T. J. Lewis, "Nanometric dielectrics," IEEE Transactions on Dielectrics and Electrical Insulation, vol. 1, no. 5, pp. 812-825, 1994.

[6] Z. Xiao, Y. Li, D. Ma, L. S. Schadler, and Y. A. Akpalu, "Probing the use of small-angle light scattering for characterizing structure of titanium dioxide/low-density polyethylene nanocomposites," Journal of Polymer Science Part B: Polymer Physics, vol. 44, no. 7, pp. 1084-1095, 2006.

[7] D. Brown, P. Mélé, S. Marceau, and N. D. Albérola, "A Molecular Dynamics Study of a Model Nanoparticle Embedded in a Polymer Matrix," Macromolecules, vol. 36, no. 4, pp. 1395-1406, 2003/02/01 2003.

[8] S. S. Sternstein and A.-J. Zhu, "Reinforcement Mechanism of Nanofilled Polymer Melts As Elucidated by Nonlinear Viscoelastic Behavior," Macromolecules, vol. 35, no. 19, pp. 7262-7273, 2002/09/01 2002.

[9] M. S. Khalil, "The role of BaTiO/sub 3/ in modifying the dc breakdown strength of LDPE," IEEE Transactions on Dielectrics and Electrical Insulation, vol. 7, no. 2, pp. 261-268, 2000.

[10] G. Chen and A. E. Davies, "The influence of defects on the short-term breakdown characteristics and long-term dc performance of LDPE insulation," IEEE Transactions on Dielectrics and Electrical Insulation, vol. 7, no. 3, pp. 401-407, 2000.

[11] T. P. Schuman, S. Siddabattuni, O. Cox, and F. Dogan, "Improved Dielectric Breakdown Strength of Covalently-Bonded Interface PolymerParticle Nanocomposites," Composite Interfaces, vol. 17, no. 8, pp. 719731, 2010/01/01 2010.

[12] S. Siddabattuni, T. P. Schuman, and F. Dogan, "Dielectric Properties of Polymer-Particle Nanocomposites Influenced by Electronic Nature of 
Filler Surfaces," ACS Applied Materials \& Interfaces, vol. 5, no. 6, pp. 1917-1927, 2013/03/27 2013.

[13] G. C. Montanari et al., "Potentiality of nanofilled thermoplastic insulation for DC cables and capacitors," in 2018 IEEE 2nd International Conference on Dielectrics (ICD), 2018, pp. 1-4.

[14] X. Wang, Z. Lv, K. Wu, X. Chen, D. Tu, and L. A. Dissado, "Study of the factors that suppress space charge accumulation in LDPE nanocomposites," IEEE Transactions on Dielectrics and Electrical Insulation, vol. 21, no. 4, pp. 1670-1679, 2014.

[15] K. Y. Lau, A. S. Vaughan, G. Chen, and I. L. Hosier, "Space charge dynamics in silica-based polyethylene nanocomposites," in 2013 IEEE International Conference on Solid Dielectrics (ICSD), 2013, pp. 880-883.

[16] B. Qiao, T.-J. Wang, H. Gao, and Y. Jin, "High density silanization of nano-silica particles using $\gamma$-aminopropyltriethoxysilane (APTES)," Applied Surface Science, vol. 351, pp. 646-654, 2015/10/01/ 2015.

[17] G. M. Sessler, Electrets. Vol. 33, Berlin, Heidelberg: Springer Berlin Heidelberg, 1987. 